Editorial

\title{
Aerospace Best Paper Awards 2018
}

\author{
Aerospace Editorial Office
}

MDPI, St. Alban-Anlage 66, 4052 Basel, Switzerland; aerospace@mdpi.com

Received: 5 March 2020; Accepted: 5 March 2020; Published: 6 March 2020

Aerospace has launched annual awards to recognize outstanding papers published in the journal.

We are pleased to announce the "Aerospace Best Paper Awards" for 2018. Nominations were chosen from all papers published in 2018, and decisions were made by the Editorial Board together with the Editorial Office. Following a review process by the Editorial Board, the following three top-voted research articles, in no particular order, have won "Aerospace Best Paper Awards" for 2018:

Single-Sensor Acoustic Emission Source Localization in Plate-Like Structures Using Deep Learning Arvin Ebrahimkhanlou and Salvatore Salamone (Figure 1)

Aerospace 2018, 5, 50; https://doi.org/10.3390/aerospace5020050

Available online: https://www.mdpi.com/2226-4310/5/2/50

Metallic panels are ubiquitous in the aerospace industry. These structures are susceptible to different types of damage, including fatigue cracks and corrosion dents. As these defects initiate and progress, they emit acoustic noises that can be used to detect and localize damage in metallic panels. To this end, this paper introduced the first deep-learning-based algorithm for localizing acoustic emission (AE) sources in metallic panels. The main idea is that deep learning can use the full length of an AE waveform and automatically extract key information pertinent to the location of AE sources. This is in contrast with the approach commonly used in most traditional AE source localization algorithms. The traditional approach relies only on the first portion of AE waveforms and does not account for the reflections and reverberations generated by the geometric features of metallic panels, such as boundaries, joints, stiffeners, and fasteners. Ignoring such reverberations makes most source localization algorithms prone to false positives, which means either incorrectly identifying the location of defects or, even worse, localizing artificial defects that do not exist in reality. To overcome that issue, this paper leveraged the reflection and reverberation patterns of AE waveforms as well as their dispersive and multimodal characteristics to localize their sources with only one sensor. Specifically, two deep-learning approaches were introduced for localizing AE sources in metallic panels with geometric features: (1) a stack of autoencoders and (2) a convolutional neural network. Experimental results showed that both deep-learning networks can learn a mapping of AE signals to their sources. These results demonstrated that the reverberation patterns of AE sources contain pertinent information to the location of their sources. 


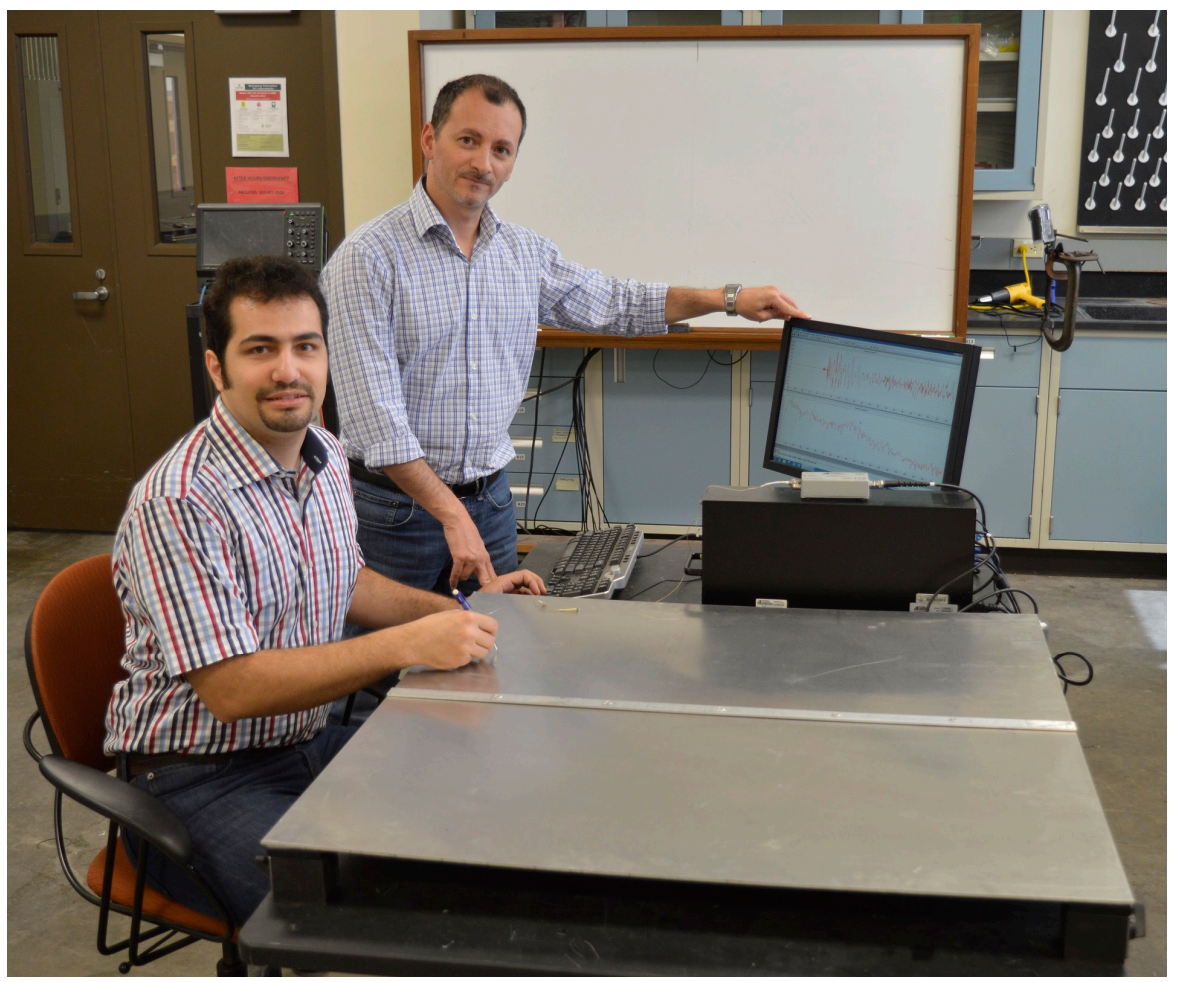

Figure 1. Dr. Ebrahimkhanlou and Dr. Salamone.

\section{Fast Aircraft Turnaround Enabled by Reliable Passenger Boarding}

Michael Schultz (Figure 2)

Aerospace 2018, 5, 8; https://doi.org/10.3390/aerospace5010008

Available online: https://www.mdpi.com/2226-4310/5/1/8

A high degree of standardization in operational processes is required to ensure efficient and effective air traffic management, during the flight stages and ground-handling stages. Deviations and disruptions in the complex and closely interlinked handling processes often lead to delays, which as the day progresses can have an increasingly negative effect on airline operations, and indeed of the entire air traffic network. Ground-handling processes can be collectively considered under the term 'turnaround' and consist of the unloading and loading of freight and baggage, refueling, cleaning, inflight catering, and the boarding and disembarking of passengers. In this context, aircraft turnaround is mainly controlled by operational experts. On the other hand, the critical aircraft-boarding process is to a large extent driven by passengers, and the speed of boarding is influenced by their prior experience of flying and their willingness or ability to follow procedures. Passengers also demand ever-greater efficiency and comfort from their travel experiences, and see non-efficient queuing at the boarding gate as a major 'pain point' in their desired seamless journey.

Perhaps the first analysis with regards to speeding up passenger boarding was provided by Marelli et al. in 1998. They compared what they regarded as the most promising approaches for boarding a single-aisle aircraft, namely the use of two doors and a concept called 'outside-in' (window seats boarded first, then middle seats, and then the aisle seats). They found that the use of a second door (a middle door) cut boarding times by $20 \%$, while the outside-in approach halved boarding times. The key philosophy behind these approaches is the predefinition of an optimal sequence for passengers entering the aircraft, a sequence which can mitigate waiting times in the aisle caused by people stowing luggage in overhead compartments, or taking time to locate and access their seats. Today, 21 years later, airlines are still researching how to make boarding more efficient, and many still consider Marelli's ideas, often in a four-year cycle. Airlines are still reinventing, testing, and implementing these 'new' 
technologies. Instead of reinventing the wheel and using methods from the last century, we should follow fundamental and applied research contributions and ask ourselves if they are the right way to push aviation into the future. Marelli et al. used computer simulations to discover and evaluate efficient boarding strategies. However, with today's mobile devices, Internet of Things, Industry 4.0, Big Data, digitization, and machine learning, we should use the tremendous potential of such state-of-the-art technologies and really pursue the motivation of Marelli's first research-gaining control of the sequence of passengers.

In this context, faster boarding does not mean passengers waiting in the jetway instead of in front of a quick-boarding gate, but rather that all passengers should be seated in the aircraft faster, and reliably faster. Successful tests of biometric scans at boarding gates have shown that such scans can shorten the time required for passenger authentication, but that this can just result in passenger queues in the jetway growing nearly $25 \%$ faster. With efficient boarding in mind, three operational questions arise: How can the queue in the jetway be reduced by faster seating? How can operators get control over the passenger sequence, dynamically? And how can current technologies support aircraft boarding? This paper provides an overview about the author's fundamental and applied research with a focus on reliable passenger boarding and innovative technologies tested in the field.

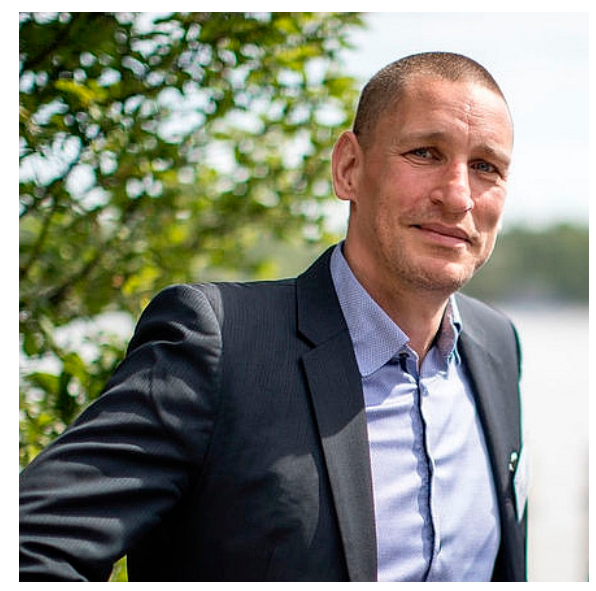

Figure 2. Dr. Schultz.

\section{Sound Absorption Characterization of Natural Materials and Sandwich Structure Composites} Jichun Zhang, Yiou Shen, Bing Jiang and Yan Li (Figure 3)

Aerospace 2018, 5, 75; https://doi.org/10.3390/aerospace5030075

Available online: https://www.mdpi.com/2226-4310/5/3/75

This paper aimed at investigating an environmentally friendly lightweight sandwich panel manufactured with flax fiber-reinforced composite skins and balsa wood core. This type of sandwich structure provides superior sound absorption performance compared to artificial fiber-based sandwich structures due to its multiscale and hollow structure, which produces more sound wave attenuation and energy dissipation. The excellent sound absorption performance of this sandwich structure enables its application in the field of vehicle or building interior structures.

We believe that these three exceptional papers are valuable contributions to Aerospace and the scientific research field. On behalf of the Aerospace Editorial Board, we would like to congratulate these teams for their excellent work. A certificate will be given to each of them.

We would like to take this opportunity to thank all the nominated research groups of the above exceptional papers for their contributions to Aerospace, and thank the Aerospace Editorial Board for voting and helping with these Best Paper Awards.

The Editorial Board and Editorial Staff at Aerospace is committed to meeting the needs of the scientific community by providing useful and timely reviews of all manuscripts submitted, and 
providing an open access forum for your results. Please consider submitting your work to Aerospace, and we look forward to announcing your paper as an Aerospace Best Paper in the future.

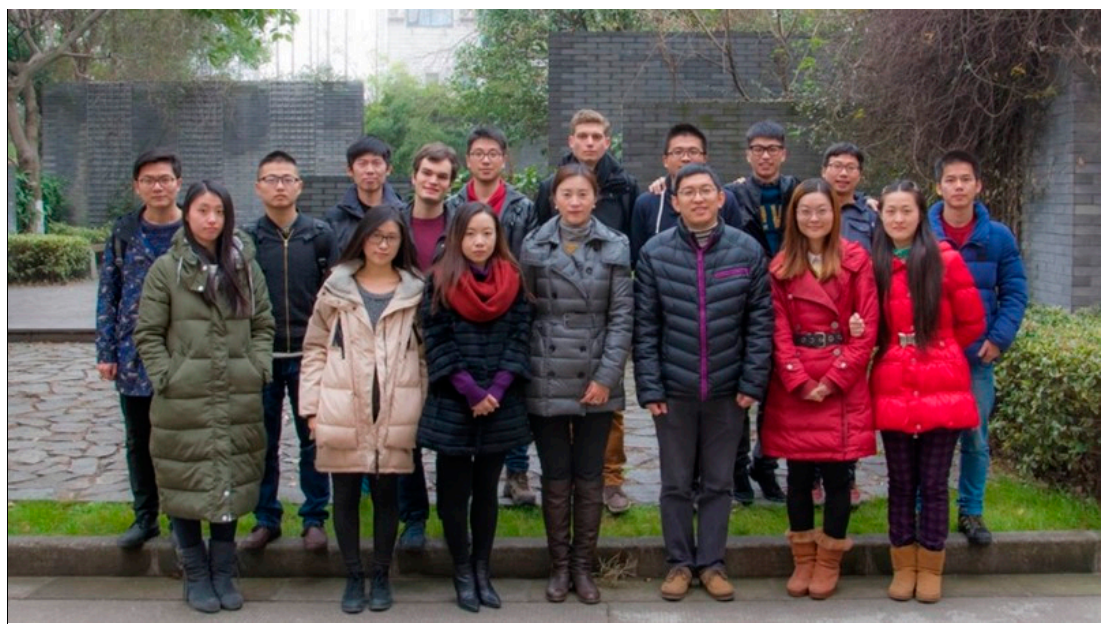

Figure 3. Professor Shen's research group.

\section{Prize Awarding Committee}

Aerospace Editorial Board

(C) 2020 by the author. Licensee MDPI, Basel, Switzerland. This article is an open access article distributed under the terms and conditions of the Creative Commons Attribution (CC BY) license (http://creativecommons.org/licenses/by/4.0/). 